\title{
A CONSTRUCTION OF INTEGRAL STANDARD GENERALIZED TABLE ALGEBRAS FROM PARAMETERS OF PROJECTIVE GEOMETRIES
}

\author{
BY \\ YASUTAKA ASABA \\ Graduate School of Technology, Shinshu University \\ Matsumoto, Nagano, 390-8621, Japan \\ AND \\ AKIHIDE HANAKI \\ Faculty of Science, Shinshu University \\ Matsumoto, Nagano, 390-8621, Japan \\ e-mail: hanaki@shinshu-u.ac.jp
}

\begin{abstract}
We construct new integral standard generalized table algebras from parameters of projective geometries. The algebras are noncommutative, imprimitive, and six dimensional.
\end{abstract}

\section{Introduction}

In [5], Zieschang and the second author investigated structures of noncommutative association schemes with 6 relations. If such an association scheme has a non-normal closed subset with two relations, then it defines a 2-design. Conversely, it was shown that some parameters of 2-designs define ISGT-algebras (integral standard generalized table algebras in the sense of [1]). But it was not determined which kind of parameters define ISGT-algebras. In this article, we will show that parameters of 2-designs obtained from projective geometries

Received December 5, 2010 and in revised form September 2, 2011 
define 6-dimensional noncommutative ISGT-algebras. Our results contain some parameters given in [5].

In Section 2, we describe a relation between association schemes and 2designs. Results in the section are based on [5] and duplicated, but written from a different point of view. In Section 3, we will construct algebras with two parameters $n$ and $q$ by the method in [5]. In Section 4, we will compute all structure constants of the algebras, and in Section 5, we will show that the algebras are ISGT-algebras if $n \geq 1$ and $q \geq 2$. In Section 6 , we will state our main result.

\section{Association schemes and 2-designs}

Results in this section are based on [5] and duplicated, but written from a different point of view. In [5], many things were written in terms of structure constants. Here we will write them in terms of parameters of 2-designs.

In this section, $(X, S)$ is a noncommutative association scheme with a nonnormal closed subset $T$ with $|S|=6$ and $|T|=2$. Then $S$ has exactly two non-symmetric relations by the Frobenius-Schur Theorem [6, (7.5) and (7.6)]. We put $S=\left\{1, t, u, w, s, s^{*}\right\}$ and $T=\{1, t\} . \operatorname{Put} \operatorname{Irr}(S)=\left\{1_{S}, \varphi, \chi\right\}$ where $\varphi(1)=1$ and $\chi(1)=2$, and put $\operatorname{Irr}(T)=\left\{1_{T}, \tau\right\}$.

Lemma 2.1: We have $1_{T}{ }^{S}=1_{S}+\chi$ and $\tau^{S}=\varphi+\chi$.

Proof. Since $T$ is non-normal in $S, 1_{T}{ }^{S}=1_{S}+\chi$ or $1_{T}{ }^{S}=1_{S}+\varphi+\chi$. Suppose that $1_{T}{ }^{S}=1_{S}+\varphi+\chi$. Then $\tau^{S}=\chi$, but this is impossible by [3, Theorem 5.1].

Lemma 2.2: We have $|S / / T|=2$.

Proof. This is clear by Lemma 2.1 and [4, Theorem 3.8].

Lemma 2.3: We may set $T u=\{u, s\}$ and $T s^{*}=\left\{s^{*}, w\right\}$.

Proof. If $T u=\{u\},\{u, w\}$, or $\left\{u, s, s^{*}\right\}$, then $T u T=T u \neq\left\{u, w, s, s^{*}\right\}$ and this contradicts Lemma 2.2. So we may put $T u=\{u, s\}$. Then $T s^{*}=$ $\left\{s^{*}, w\right\}$.

2.1. A 2-DESign from An ASSOCiATion SCheme. In this subsection, we will construct a 2-design from an association scheme with the above properties. 
Lemma 2.4: For $x, x^{\prime}, y, y^{\prime} \in X$, assume that $y \in x u, y^{\prime} \in x^{\prime} u, x^{\prime} \in x T$, and $y^{\prime} \in y T$. Then $y^{\prime} \in x u$.

Proof. If $x=x^{\prime}$ or $y=y^{\prime}$, then the statement is clear.

Assume that $x \neq x^{\prime}$ and $y \neq y^{\prime}$. Then $x^{\prime} \in x t$ and $y^{\prime} \in y t$. Now $y^{\prime} \in$ $x^{\prime} u \cap y t \subseteq x t u \cap x u t=x(t u \cap u t)=x u$.

Let

$$
X=x_{0} T \cup x_{1} T \cup \cdots \cup x_{b} T
$$

be a coset decomposition of $X$. Put

$$
P=x_{0} T \text {. }
$$

For $i=1,2, \ldots, b$, we define

$$
B_{i}=\left\{p \in P \mid p u \cap x_{i} T \neq \emptyset\right\} .
$$

Put $\mathcal{B}=\left\{B_{i} \mid i=1,2, \ldots, b\right\}$.

Theorem 2.5: The incidence structure $(P, \mathcal{B}, \in)$ is a $2-(v, k, \lambda)$ design where $(v, k, \lambda)=\left(n_{t}+1, a_{u t u}+1, a_{u u t} /\left(a_{u t u}+1\right)\right)$.

Proof. Since $|S| / T \mid=2,\left(P \times x_{i} T\right) \cap u \neq \emptyset$. Fix $p \in P$ and $x \in x_{i} T \cap p u$. Then $B_{i}=x u \cap P$. Now $B_{i}=\{p\} \cup(x u \cap p t)$. We have that $\left|B_{i}\right|=a_{u t u}+1$ and this number is independent of the choice of $i$. Put

$$
k=\left|B_{i}\right|=a_{u t u}+1 .
$$

If $p^{\prime} \in B_{i}$, then $\left|p^{\prime} u \cap x_{i} T\right|=k$. Thus $\left|\left(P \times x_{i} T\right) \cap u\right|=k^{2}$ for every $i \in\{1,2, \ldots, b\}$.

Fix $p \in P$ and $q \in P$ such that $p \neq q$. Easily we can see that $p u \cap q u \cap x_{i} T \neq \emptyset$ if and only if $\{p, q\} \subseteq B_{i}$. Put

$$
\lambda=\left|\left\{i \mid\{p, q\} \subseteq B_{i}\right\}\right|=\left|\left\{i \mid p u \cap q u \cap x_{i} T \neq \emptyset\right\}\right| .
$$

Note that, if $p u \cap q u \cap x_{i} T \neq \emptyset$, then $p u \cap q u \cap x_{i} T=p u \cap x_{i} T=q u \cap x_{i} T$ by Lemma 2.4 and in this case $\left|p u \cap q u \cap x_{i} T\right|=k$. Now

$$
a_{u u t}=|p u \cap q u|=\sum_{i=1}^{b}\left|p u \cap q u \cap x_{i} T\right| .
$$

So we have that

$$
a_{u u t}=k \lambda \text {. }
$$

This means that $\lambda$ is constant. 
Now $(P, \mathcal{B}, \in)$ is a $2-(v, k, \lambda)$ design, where $v=n_{T}=1+n_{t}$.

Remark 2.1: The 2-design obtained in Lemma 2.5 can have repeated blocks and allows the case $\lambda=0$. Also we remark that, if we change the base point, then the obtained 2-designs may be non-equivalent. But their parameters are the same.

For $i=1,2, \ldots, b$, we define

$$
B_{i}^{\prime}=\left\{p \in P \mid p w \cap x_{i} T \neq \emptyset\right\}
$$

and $\mathcal{B}^{\prime}=\left\{B_{1}^{\prime}, \ldots, B_{b}^{\prime}\right\}$. Then $\left(P, \mathcal{B}^{\prime}, \in\right)$ is the complement of $(P, \mathcal{B}, \in)$. So it is a $2-(v, v-k, b-2 r+\lambda)$ design, where $b$ and $r$ are parameters of the a 2-design $(P, \mathcal{B}, \in)$ determined by well-known equations $b k=v r$ and $r(k-1)=\lambda(v-1)$.

2.2. Intersection NUMBERS FROM PARAMETERS OF A 2-DESIGN. In the previous subsection, we constructed a 2-design from an association scheme. The parameters of a 2-design are determined by the intersection numbers of the scheme. In this section, we compute intersection numbers from parameters of a 2-design. Of course, all intersection numbers must be non-negative integers. So the parameters must satisfy very strong conditions. It seems to be difficult to determine all such parameters. We will give some examples of parameters which give non-negative integer intersection numbers. We do not know if they come from an association scheme, but we can construct integral table algebras.

Suppose that a 2-design is obtained as in the previous subsection. Let $(v, k, \lambda, b, r)$ be the parameters of the 2-design. We know that

$$
b k=v r, \quad r(k-1)=\lambda(v-1)
$$

LEMmA 2.6: (1) $a_{t u u}+1=a_{t u s}, a_{t s s}+1=a_{t s u}, a_{t s^{*} s^{*}}+1=a_{t s^{*} w}$, and $a_{t w w}+1=a_{t w s^{*}}$.

(2) $k=a_{u t u}+1=a_{\text {tuu }}+1=a_{\text {tus }}=a_{u t s^{*}}$ and $\lambda k=a_{u u t}$.

(3) $n_{t}=v-1, n_{u}=r k, n_{s}=n_{s^{*}}=(v-k) r$, and $n_{w}=\frac{(v-k)^{2} r}{k}$.

Proof. (1) is by [9, Lemma 2.3.1]. (2) and (3) are by the proof of Lemma 2.5 .

Recall that $\varphi$ is the non-trivial irreducible character of $S$ of degree 1. 
LEMma 2.7: Let $\varphi$ be the non-trivial irreducible character of $S$ of degree one. Then we have $\varphi\left(\sigma_{t}\right)=-1$ and $\varphi\left(\sigma_{u}\right)=-\varphi\left(\sigma_{s}\right)=-\varphi\left(\sigma_{s^{*}}\right)=\varphi\left(\sigma_{w}\right)$.

Proof. Since $\left.\varphi\right|_{T}$ is a non-trivial character, we have $\varphi\left(\sigma_{t}\right)=-1$. Note that $\varphi\left(\sigma_{u}\right)$ is a real number since $u$ is a symmetric relation. By $\sigma_{t} \sigma_{u}=(k-1) \sigma_{u}+k \sigma_{s}$, $\varphi\left(\sigma_{u}\right)=-\varphi\left(\sigma_{s}\right)$ holds. Similarly, by $\sigma_{t} \sigma_{s^{*}}=(k-1) \sigma_{s^{*}}+k \sigma_{w}, \varphi\left(\sigma_{s^{*}}\right)=-\varphi\left(\sigma_{w}\right)$ holds. So $\varphi\left(\sigma_{s}\right)$ is a real number and $\varphi\left(\sigma_{s}\right)=\varphi\left(\sigma_{s^{*}}\right)$ holds.

We give multiplicities of irreducible characters.

Lemma 2.8: We have $m_{\varphi}=b v+v-2 b-1$ and $m_{\chi}=b$.

Proof. Since $1_{T}{ }^{S}=1_{S}+\chi, m_{\chi}=n_{S} / n_{T}-1=b$ by [3, Theorem 5.1]. The equation on $m_{\varphi}$ is obtained by $\sum_{\xi \in \operatorname{Irr}(S)} m_{\xi} \xi(1)=n_{S}$.

The next lemma is [5, Theorem 2.4.3] and not so easy to prove and not crucial in our arguments. So we omit the proof.

LEMMA 2.9: If $k=1$ or $k=v-1$, then the scheme is a Coxeter scheme or a semidirect product of smaller schemes.

From now on, we assume that $k>1$. Then

$$
r=\frac{\lambda(v-1)}{k-1}, \quad b=\frac{\lambda v(v-1)}{k(k-1)} .
$$

Let $\Psi: \mathbb{C} S \rightarrow M_{2}(\mathbb{C})$ be a representation of $S$ affording $\chi$. By Lemma 2.1, we have $\chi_{T}=1_{T}+\tau$. So we may assume that

$$
\Psi\left(\sigma_{t}\right)=\left(\begin{array}{cc}
v-1 & 0 \\
0 & -1
\end{array}\right)
$$

Set

$$
\Psi\left(\sigma_{u}\right)=\left(\begin{array}{ll}
u_{11} & u_{12} \\
u_{21} & u_{22}
\end{array}\right)
$$

By Lemma 2.3 and Lemma 2.6, we have $\sigma_{t} \sigma_{u}=(k-1) \sigma_{u}+k \sigma_{s}, \sigma_{u} \sigma_{t}=$ $(k-1) \sigma_{u}+k \sigma_{s^{*}}$, and $\sigma_{s} \sigma_{t}=(k-1) \sigma_{s}+k \sigma_{w}$. So we have

$$
\Psi\left(\sigma_{s}\right)=\left(\begin{array}{cc}
\frac{v-k}{k} u_{11} & \frac{v-k}{k} u_{12} \\
-u_{21} & -u_{22}
\end{array}\right), \quad \Psi\left(\sigma_{s^{*}}\right)=\left(\begin{array}{cc}
\frac{v-k}{k} u_{11} & -u_{12} \\
\frac{v-k}{k} u_{21} & -u_{22}
\end{array}\right),
$$

and

$$
\Psi\left(\sigma_{w}\right)=\left(\begin{array}{cc}
\frac{(v-k)^{2}}{k^{2}} u_{11} & -\frac{v-k}{k} u_{12} \\
-\frac{v-k}{k} u_{21} & u_{22}
\end{array}\right)
$$


By $\sum_{\ell \in S} \Psi\left(\sigma_{\ell}\right)=0$, we have

$$
u_{11}=-\frac{k^{2}}{v}
$$

By the Schur relations [9, Theorem 9.2.4] to the $(1,2)$-entries, we have

$$
u_{12} u_{21}=\frac{k^{2}(v-k)\left(\lambda v^{2}-\lambda v+k^{2}-k\right)}{v^{2}(k-1)} .
$$

We note that $u_{12} u_{21} \neq 0$ since $\Psi$ is irreducible. So we can change $u_{12}$ and $u_{21}$ keeping $u_{12} u_{21}$ by considering a similar representation. For example, we may assume that $u_{21}=1$. Again by the Schur relations to the $(2,2)$-entries, we have

$$
u_{22}^{2}=\frac{k(v-k)^{2}\left(\lambda v^{2}-2 \lambda v+k^{2}-k\right)}{v^{2}(k-1)} .
$$

So

$$
u_{22}=\varepsilon \frac{v-k}{v} \sqrt{\frac{k\left(\lambda v^{2}-2 \lambda v+k^{2}-k\right)}{k-1}}
$$

for $\varepsilon \in\{ \pm 1\}$. Now we can determine $\Psi\left(\sigma_{\ell}\right)$ for all $\ell \in S$ by the parameters of the 2 -design and a $\operatorname{sign} \varepsilon$. Hence we get character values of $\chi$.

Now we can also compute the values of $\varphi$. Then by the formula

$$
a_{x y z}=\frac{1}{n_{z}|X|} \sum_{\xi \in \operatorname{Irr}(S)} m_{\xi} \xi\left(\sigma_{x} \sigma_{y} \sigma_{z^{*}}\right),
$$

we can compute all intersection numbers. Here, to compute $\chi\left(\sigma_{a} \sigma_{b} \sigma_{c^{*}}\right)$, we use the matrix trace of $\Psi\left(\sigma_{a}\right) \Psi\left(\sigma_{b}\right) \Psi\left(\sigma_{c^{*}}\right)$.

General forms of intersection numbers are very complicated. So we do not compute them here and will compute them for some special cases.

\section{Construction}

Let $n$ and $q$ be integers greater than 1 . We consider a projective geometry $\mathrm{PG}_{n-1}(n, q)$ (see [2, I, Definition 2.15, Proposition 2.16]). It defines a 2-design of parameters

$$
\left(v, k, \lambda^{\prime}\right)=\left(\frac{q^{n+1}-1}{q-1}, \frac{q^{n}-1}{q-1}, \frac{q^{n-1}-1}{q-1}\right) .
$$

In this article, we consider a 2 -design obtained by $q k$-times repeating of $\mathrm{PG}_{n-1}(n, q)$. So the parameters are

$$
(v, k, \lambda)=\left(\frac{q^{n+1}-1}{q-1}, \frac{q^{n}-1}{q-1}, \frac{q\left(q^{n}-1\right)\left(q^{n-1}-1\right)}{(q-1)^{2}}\right) .
$$


We remark that $q$ must be a prime power to define $\mathrm{PG}_{n-1}(n, q)$. But in this article, we only use the parameters and $q$ is not necessarily a prime power.

By the method in [5] or Subsection 2.2, we will define a 6 -dimensional $\mathbb{C}$ algebra $A$ with basis $\mathbf{B}=\left\{1, t, u, s, s^{*}, w\right\}$. Make a two-dimensional irreducible representation by the system of parameters above as in Subsection 2.2, and consider the direct sum of three irreducible representations. Then the algebra $A$ will be defined as a matrix algebra of degree 4 . We fix a $\operatorname{sign} \varepsilon$ and get

$$
\begin{aligned}
& 1=\left(\begin{array}{llll}
1 & 0 & 0 & 0 \\
0 & 1 & 0 & 0 \\
0 & 0 & 1 & 0 \\
0 & 0 & 0 & 1
\end{array}\right) \\
& t=\left(\begin{array}{cccc}
\frac{q\left(q^{n}-1\right)}{q-1} & 0 & 0 & 0 \\
0 & -1 & 0 & 0 \\
0 & 0 & \frac{q\left(q^{n}-1\right)}{q-1} & 0 \\
0 & 0 & 0 & -1
\end{array}\right) \\
& u=\left(\begin{array}{cccc}
\frac{q\left(q^{n}-1\right)^{3}}{(q-1)^{3}} & 0 & 0 & 0 \\
0 & -q^{n-1} & 0 & 0 \\
0 & 0 & -\frac{\left(q^{n}-1\right)^{2}}{(q-1)\left(q^{n+1}-1\right)} & \frac{q^{n}\left(q^{n}-1\right)^{3}\left(q^{2 n+2}-q^{n+2}-q^{n+1}+q^{2}-q+1\right)}{(q-1)^{3}\left(q^{n+1}-1\right)^{2}} \\
0 & 0 & 1 & \frac{q^{n+1}\left(q^{n}-1\right)^{2}}{(q-1)\left(q^{n+1}-1\right)}
\end{array}\right), \\
& s=\left(\begin{array}{cccc}
\frac{q^{n+1}\left(q^{n}-1\right)^{2}}{(q-1)^{2}} & 0 & 0 & 0 \\
0 & q^{n-1} & 0 & 0 \\
0 & 0 & -\frac{q^{n}\left(q^{n}-1\right)}{q^{n+1}-1} & \frac{q^{2 n}\left(q^{n}-1\right)^{2}\left(q^{2 n+2}-q^{n+2}-q^{n+1}+q^{2}-q+1\right)}{(q-1)^{2}\left(q^{n+1}-1\right)^{2}} \\
0 & 0 & -1 & -\frac{q^{n+1}\left(q^{n}-1\right)^{2}}{(q-1)\left(q^{n+1}-1\right)}
\end{array}\right), \\
& s^{*}=\left(\begin{array}{cccc}
\frac{q^{n+1}\left(q^{n}-1\right)^{2}}{(q-1)^{2}} & 0 & 0 & 0 \\
0 & q^{n-1} & 0 & 0 \\
0 & 0 & -\frac{q^{n}\left(q^{n}-1\right)}{q^{n+1}-1} & -\frac{q^{n}\left(q^{n}-1\right)^{3}\left(q^{2 n+2}-q^{n+2}-q^{n+1}+q^{2}-q+1\right)}{(q-1)^{3}\left(q^{n+1}-1\right)^{2}} \\
0 & 0 & \frac{q^{n}(q-1)}{q^{n}-1} & -\frac{q^{n+1}\left(q^{n}-1\right)^{2}}{(q-1)\left(q^{n+1}-1\right)}
\end{array}\right) \text {, } \\
& w=\left(\begin{array}{cccc}
\frac{q^{2 n+1}\left(q^{n}-1\right)}{q-1} & 0 & 0 & 0 \\
0 & -q^{n-1} & 0 & 0 \\
0 & 0 & -\frac{q^{2 n}(q-1)}{q^{n+1}-1} & -\frac{q^{2 n}\left(q^{n}-1\right)^{2}\left(q^{2 n+2}-q^{n+2}-q^{n+1}+q^{2}-q+1\right)}{(q-1)^{2}\left(q^{n+1}-1\right)^{2}} \\
0 & 0 & -\frac{q^{n}(q-1)}{q^{n}-1} & \frac{q^{n+1}\left(q^{n}-1\right)^{2}}{(q-1)\left(q^{n+1}-1\right)}
\end{array}\right) .
\end{aligned}
$$


In the rest of this article, we will only use these matrices and show that $(A, \mathbf{B})$ is an ISGT-algebra [1]. For any $x \in \mathbf{B}$, we can write

$$
x=\left(\begin{array}{lll}
T_{1}(x) & & \\
& T_{2}(x) & \\
& & T_{3}(x)
\end{array}\right)
$$

where $T_{i}(i=1,2,3)$ are all irreducible representations of the algebra $A$. We can see that

$$
A \cong \mathbb{C} \oplus \mathbb{C} \oplus M_{2}(\mathbb{C})
$$

and $A$ is noncommutative.

\section{Structure constants}

In this section, we compute all structure constants $\left\{a_{x y z} \mid x, y, z \in \mathbf{B}\right\}$ of the algebra $A$ with respect to the basis $\mathbf{B}=\left\{1, t, u, s, s^{*}, w\right\}$. Namely, for $x, y, z \in \mathbf{B}$,

$$
x y=\sum_{z \in \mathbf{B}} a_{x y z} z .
$$

Theoretically, it is possible to compute all structure constants directly by the matrices. But it is not so easy even if we use computers. If the algebra is obtained as an adjacency algebra of an association scheme $(X, S)$ (in the sense of [8]), then we can use a useful formula

$$
a_{x y z}=\frac{1}{n_{z}|X|} \sum_{\chi \in \operatorname{Irr}(S)} m_{\chi} \chi\left(\sigma_{x} \sigma_{y} \sigma_{z^{*}}\right)
$$

on association schemes. We do not explain details, but we use this formula to compute structure constants $a_{x y z}(x, y, z \in \mathbf{B})$. After that we need to check that $a_{x y z}$ obtained in this way are really structure constants of the algebra, because we do not know there is an association scheme.

All computations were done by Maxima [7]. Let $L: A \rightarrow M_{6}(\mathbb{C})$ be the left regular representation of $A$ with respect to the basis B. Namely $L(x)$ is a matrix with the $(z, y)$-entry $a_{x y z}$. We get the following matrices of structure 
Vol. 194, 2013

constants:

$$
L(1)=\left(\begin{array}{llllll}
1 & 0 & 0 & 0 & 0 & 0 \\
0 & 1 & 0 & 0 & 0 & 0 \\
0 & 0 & 1 & 0 & 0 & 0 \\
0 & 0 & 0 & 1 & 0 & 0 \\
0 & 0 & 0 & 0 & 1 & 0 \\
0 & 0 & 0 & 0 & 0 & 1
\end{array}\right)
$$

$$
L(t)=\left(\begin{array}{cccccc}
0 & \frac{q\left(q^{n}-1\right)}{q-1} & 0 & 0 & 0 & 0 \\
1 & \frac{q^{n+1}-2 q+1}{q-1} & 0 & 0 & 0 & 0 \\
0 & 0 & \frac{q^{n}-q}{q-1} & q^{n} & 0 & 0 \\
0 & 0 & \frac{q^{n}-1}{q-1} & q^{n}-1 & 0 & 0 \\
0 & 0 & 0 & 0 & \frac{q^{n}-q}{q-1} & q^{n} \\
0 & 0 & 0 & 0 & \frac{q^{n}-1}{q-1} & q^{n}-1
\end{array}\right),
$$

$$
\begin{aligned}
L(u)= & \left(\begin{array}{cccc}
0 & 0 & \frac{q\left(q^{n}-1\right)^{3}}{(q-1)^{3}} \\
0 & 0 & \frac{\left(q^{n}-1\right)^{2}\left(q^{n}-q\right)}{(q-1)^{3}} \\
1 & \frac{q^{n}-q}{q-1} & \frac{q^{2 n+3}-q^{2 n+2}-3 q^{2 n+1}-3 q^{n+3}+7 q^{n+2}-q^{n+1}+q^{3 n}+q^{3}-3 q^{2}+q}{(q-1)^{3} q} \\
0 & 0 & \frac{\left(q^{n}-1\right)\left(q^{n}-q\right)^{2}}{(q-1)^{3} q} \\
0 & \frac{q^{n}-1}{q-1} & \frac{\left.\left(q^{n}-1\right) q^{n}-q\right)^{2}}{(q-1)^{3} q} \\
0 & 0 & \frac{\left(q^{n}-1\right)^{2}\left(q^{n}-q\right)}{(q-1)^{3} q} & \\
0 & 0 & 0 \\
\frac{q^{n-1}\left(q^{n}-q\right)^{2}}{(q-1)^{2}} & \frac{q^{n}\left(q^{n}-1\right)^{2}}{(q-1)^{2}} & 0 \\
\frac{q^{n-1}\left(q^{n}-1\right)\left(q^{n}-q\right)}{(q-1)^{2}} & \frac{q^{n-1}\left(q^{n}-1\right)^{2}}{\left.(q-1) q^{n}-q\right)} & \frac{q^{2 n-1}\left(q^{n}-q\right)}{q-1} \\
(q-1)^{2} & \frac{q^{2 n-1}\left(q^{n}-1\right)}{q-1} \\
\frac{q^{n-1}\left(q^{n}-1\right)^{2}}{(q-1)^{2}} & \frac{\left(q^{n}-1\right)\left(q^{n+2}-2 q^{n+1}+q^{2 n}-q^{2}+q\right)}{(q-1)^{2} q} & \frac{q^{n-1}\left(q^{n}-1\right)\left(q^{n}-q\right)}{q-1} \\
(q-1)^{2} q & \frac{q^{n-1}\left(q^{n}-1\right)^{2}}{q-1}
\end{array}\right.
\end{aligned}
$$




$$
\begin{aligned}
& L(s)=\left(\begin{array}{cccc}
0 & 0 & 0 & 0 \\
0 & 0 & \frac{q^{n}\left(q^{n}-1\right)^{2}}{(q-1)^{2}} & 0 \\
0 & 0 & \frac{q^{n-1}\left(q^{n}-q\right)^{2}}{(q-1)^{2}} & \frac{q^{2 n-1}\left(q^{n}-q\right)}{q-1} \\
1 & \frac{q^{n}-q}{q-1} & \frac{\left(q^{n}-1\right)\left(q^{n+2}-2 q^{n+1}+q^{2 n}-q^{2}+q\right)}{(q-1)^{2} q} & \frac{q^{n-1}\left(q^{n}-1\right)\left(q^{n}-q\right)}{q-1} \\
0 & 0 & \frac{q^{n-1}\left(q^{n}-1\right)\left(q^{n}-q\right)}{(q-1)^{2}} & \frac{q^{2 n-1}\left(q^{n}-1\right)}{q-1} \\
0 & \frac{q^{n}-1}{q-1} & \frac{\left(q^{n}-1\right)^{2}\left(q^{n}-q\right)}{(q-1)^{2} q} & \frac{q^{n-1}\left(q^{n}-1\right)^{2}}{q-1}
\end{array}\right. \\
& \left.\begin{array}{cc}
\frac{q^{n+1}\left(q^{n}-1\right)^{2}}{(q-1)^{2}} & 0 \\
\frac{q^{n}\left(q^{n}-1\right)^{2}}{q-1} & 0 \\
\frac{q^{2 n-1}\left(q^{n}-q\right)}{q-1} & q^{3 n-1} \\
\frac{q^{n-1}\left(q^{n}-1\right)\left(q^{n}-q\right)}{q-1} & q^{2 n-1}\left(q^{n}-1\right) \\
\frac{q^{n-1}\left(q^{n}-1\right)\left(q^{n}-q\right)}{q-1} & q^{2 n-1}\left(q^{n}-1\right) \\
\frac{\left(q^{n}-1\right)\left(q^{2 n+1}-q^{2 n}+q^{n}-q^{2}\right)}{(q-1)^{2} q} & q^{n-1}\left(q^{n}-1\right)^{2}
\end{array}\right),
\end{aligned}
$$

$$
\begin{aligned}
& L\left(s^{*}\right)=\left(\begin{array}{cccc}
0 & 0 & 0 & \frac{q^{n+1}\left(q^{n}-1\right)^{2}}{(q-1)^{2}} \\
0 & 0 & 0 & \frac{q^{n}\left(q^{n}-1\right)\left(q^{n}-q\right)}{(q-1)^{2}} \\
0 & q^{n} & \frac{q^{n-1}\left(q^{n}-q\right)^{2}}{(q-1)^{2}} & \frac{q^{n-1}\left(q^{n+2}-2 q^{n+1}+q^{2 n}-q^{2}+q\right)}{q-1} \\
0 & 0 & \frac{q^{n-1}\left(q^{n}-1\right)\left(q^{n}-q\right)}{(q-1)^{2}} & \frac{q^{n-1}\left(q^{n}-1\right)\left(q^{n}-q\right)}{q-1} \\
1 & q^{n}-1 & \frac{q^{n-1}\left(q^{n}-1\right)\left(q^{n}-q\right)}{(q-1)^{2}} & \frac{q^{n-1}\left(q^{n}-1\right)\left(q^{n}-q\right)}{q-1} \\
0 & 0 & \frac{q^{n-1}\left(q^{n}-1\right)^{2}}{(q-1)^{2}} & \frac{q^{n-1}\left(q^{n}-1\right)^{2}}{q-1}
\end{array}\right. \\
& \left.\begin{array}{cc}
0 & 0 \\
0 & \frac{q^{2 n}\left(q^{n}-1\right)}{q-1} \\
\frac{q^{2 n-1}\left(q^{n}-q\right)}{q-1} & q^{2 n-1}\left(q^{n}-q\right) \\
\frac{q^{2 n-1}\left(q^{n}-1\right)}{q-1} & q^{2 n-1}\left(q^{n}-1\right) \\
\frac{q^{n-1}\left(q^{n}-1\right)\left(q^{n}-q\right)}{q-1} & \frac{q^{n-1}\left(q^{2 n+1}-q^{2 n}+q^{n}-q^{2}\right)}{q-1} \\
\frac{q^{n-1}\left(q^{n}-1\right)^{2}}{q-1} & q^{n-1}\left(q^{n}-1\right)^{2}
\end{array}\right)
\end{aligned}
$$




$$
\begin{aligned}
L(w)= & \left(\begin{array}{cccc}
0 & 0 & 0 & 0 \\
0 & 0 & 0 & \frac{q^{2 n}\left(q^{n}-1\right)}{q-1} \\
0 & 0 & \frac{q^{2 n-1}\left(q^{n}-q\right)}{q-1} & q^{2 n-1}\left(q^{n}-q\right) \\
0 & q^{n} & \frac{q^{n-1}\left(q^{n}-1\right)\left(q^{n}-q\right)}{q-1} & \frac{q^{n-1}\left(q^{2 n+1}-q^{2 n}+q^{n}-q^{2}\right)}{q-1} \\
0 & 0 & \frac{q^{2 n-1}\left(q^{n}-1\right)}{q-1} & q^{2 n-1}\left(q^{n}-1\right) \\
1 & q^{n}-1 & \frac{q^{n-1}\left(q^{n}-1\right)^{2}}{q-1} & q^{n-1}\left(q^{n}-1\right)^{2} \\
0 & & \frac{q^{2 n+1}\left(q^{n}-1\right)}{q-1} \\
0 & & (q-1) q^{3 n-1}\left(q^{n}-1\right) \\
q^{3 n-1} & (q-1) q^{2 n-1}\left(q^{n}-1\right) \\
q^{2 n-1}\left(q^{n}-1\right) & (q-1) q^{2 n-1}\left(q^{n}-1\right) \\
q^{2 n-1}\left(q^{n}-1\right) & \frac{q^{n-1}\left(q^{2 n+2}-2 q^{2 n+1}-q^{n+2}+4 q^{n+1}+q^{2 n}-2 q^{n}-q^{2}-q+1\right)}{q-1}
\end{array}\right) \\
q^{n-1}\left(q^{n}-1\right)^{2} &
\end{aligned}
$$

\section{ISGT-algebras}

In this section, we will show that $(A, \mathbf{B})$ is an ISGT-algebra. By the table of structure constants, we can check many properties required by $A$ to be an ISGT-algebra. The representation $T_{1}$ defined in Section 3 is the map required by $(A, \mathbf{B})$ to be standard. The map ${ }^{*}: \mathbf{B} \rightarrow \mathbf{B}$ is $s \mapsto s^{*}, s^{*} \mapsto s$, and $x \mapsto x$ for $x \in\{1, t, u, w\}$. It is not so easy to check that ${ }^{*}$ defines an antiisomorphism of $A$. We need to check that $a_{x y z}=a_{y^{*} x^{*} z^{*}}$ for all $x, y, x \in \mathbf{B}$. But we can do it by the forms of $L(x)(x \in \mathbf{B})$. Now, it is enough to check that all structure constants are non-negative integers. We suppose that $n \geq 1$ and $q \geq 2$. We note that, if we use the arguments in Subsection 2.2, we need $k=\left(q^{n}-1\right) /(q-1) \geq 2$ and this means $n \geq 2$. But after we define 4-dimensional matrices, we may consider the case $n=1$. This is just the case in Lemma 2.9. Except for the following three values, it is easy to see that the entries in $L(x)$ $(x \in \mathbf{B})$ are non-negative integers.

(1) $q^{n+2}-2 q^{n+1}+q^{2 n}-q^{2}+q \geq 0$ for $a_{u s^{*} s^{*}}, a_{s^{*} s u}$, and $a_{\text {sus }}$.

(2) $q^{2 n+3}-q^{2 n+2}-3 q^{2 n+1}-3 q^{n+3}+7 q^{n+2}-q^{n+1}+q^{3 n}+q^{3}-3 q^{2}+q \geq 0$ and divided by $(q-1)^{3}$ for $a_{u u u}$.

(3) $q^{2 n+2}-2 q^{2 n+1}-q^{n+2}+4 q^{n+1}+q^{2 n}-2 q^{n}-q^{2}-q+1 \geq 0$ for $a_{w w w}$. Lemma 5.1: If $q \geq 2$ and $n \geq 1$, then $q^{n+2}-2 q^{n+1}+q^{2 n}-q^{2}+q \geq 0$. 
Proof. We have $q^{n+2}-2 q^{n+1}+q^{2 n}-q^{2}+q=\left(q^{n+1}-q\right)(q-1)+\left(q^{2 n}-q^{n+1}\right) \geq$ 0 .

LEMMA 5.2: If $q \geq 2$ and $n \geq 1$, then $q^{2 n+3}-q^{2 n+2}-3 q^{2 n+1}-3 q^{n+3}+7 q^{n+2}-$ $q^{n+1}+q^{3 n}+q^{3}-3 q^{2}+q \geq 0$ and divided by $(q-1)^{3}$.

Proof. Put $f(x)=x^{2 n+3}-x^{2 n+2}-3 x^{2 n+1}-3 x^{n+3}+7 x^{n+2}-x^{n+1}+x^{3 n}+$ $x^{3}-3 x^{2}+x$. It is easy to see that $f(1)=f^{\prime}(1)=f^{\prime \prime}(1)=0$. This means that $(x-1)^{3}$ divides $f(x)$.

Suppose $q=2$. Then $f(2)=\left(2^{2 n}+1\right)\left(2^{n}-1\right) \geq 0$.

Suppose $q \geq 3$. Then

$$
\begin{aligned}
f(q)= & q^{3 n}+\left(q\left(q^{2}-q-3\right)\right) q^{2 n}+\left(q\left(-3 q^{2}+7 q-1\right)\right) q^{n} \\
& +q\left(q^{2}-3 q+1\right) .
\end{aligned}
$$

Since $q \geq 3, q^{2}-q-3 \geq 0$. On replacing $q^{2 n}$ by $3 q^{n}$, we have

$$
f(q) \geq q^{3 n}+(q(4 q-10)) q^{n}+q\left(q^{2}-3 q+1\right) .
$$

Since $q \geq 3,4 q-10 \geq 0$ and $q^{2}-3 q+1 \geq 0$. So we have $f(q) \geq 0$.

Lemma 5.3: If $q \geq 2$ and $n \geq 1$, then

$$
q^{2 n+2}-2 q^{2 n+1}-q^{n+2}+4 q^{n+1}+q^{2 n}-2 q^{n}-q^{2}-q+1 \geq 0 .
$$

Proof. Put $f(x)=x^{2 n+2}-2 x^{2 n+1}-x^{n+2}+4 x^{n+1}+x^{2 n}-2 x^{n}-x^{2}-x+1$.

Suppose $q=2$. Then $f(2)=\left(2^{n}+1\right)^{2}-6 \geq 0$.

Suppose $q \geq 3$. Then

$$
f(q)=\left(q^{2}-2 q+1\right) q^{2 n}+\left(-q^{2}+4 q-2\right) q^{n}+\left(-q^{2}-q+1\right) .
$$

Since $q^{2}-2 q+1 \geq 0$, on replacing $q^{2 n}$ by $2 q^{n}$, we have

$$
f(q) \geq q^{n+2}-q^{2}-q+1 .
$$

On replacing $q^{n+2}$ by $2 q^{2}$, we have

$$
f(q) \geq q^{2}-q+1 .
$$

Since $q \geq 3$, we have $f(q) \geq 0$.

Now we can say that $(A, \mathbf{B})$ is an ISGT-algebra. 


\section{Main result}

Now we write our main result.

Theorem 6.1: Let $n$ and $q$ be integers with $n \geq 1$ and $q \geq 2$. Then the algebra $A$ with distinguished basis $\mathbf{B}=\left\{1, t, u, s, s^{*}, w\right\}$ defined in Section 3 is an ISGT-algebra with the following properties.

(1) $\operatorname{dim}_{\mathbb{C}} A=6$.

(2) $A$ is noncommutative.

(3) $\mathbf{C}=\{1, t\}$ is closed.

(4) The character table of $A$ is given by the following.

\begin{tabular}{c|cccccc} 
& 1 & $t$ & $u$ & $s$ & $s^{*}$ & $w$ \\
\hline$T_{1}$ & 1 & $\frac{q\left(q^{n}-1\right)}{q-1}$ & $\frac{q\left(q^{n}-1\right)^{3}}{(q-1)^{3}}$ & $\frac{q^{n+1}\left(q^{n}-1\right)^{2}}{(q-1)^{2}}$ & $\frac{q^{n+1}\left(q^{n}-1\right)^{2}}{(q-1)^{2}}$ & $\frac{q^{2 n+1}\left(q^{n}-1\right)}{q-1}$ \\
$T_{2}$ & 1 & -1 & $-q^{n-1}$ & $q^{n-1}$ & $q^{n-1}$ & $-q^{n-1}$ \\
$T_{3}$ & 2 & $\frac{q^{n+1}-2 q+1}{q-1}$ & $\frac{\left(q^{n}-1\right)^{2}}{q-1}$ & $-\frac{q^{n}\left(q^{n}-1\right)}{q-1}$ & $-\frac{q^{n}\left(q^{n}-1\right)}{q-1}$ & $\frac{q^{n}\left(q^{n}-q\right)}{q-1}$
\end{tabular}

Proof. (3) is by the form of $L(t)$ in Section 4. (4) is by the definition of 4dimensional matrices defined in Section 3.

ACKNowledGMENT. The authors would like to thank the anonymous referee for his/her careful reading and helpful comments.

\section{References}

[1] Z. Arad, E. Fisman and M. Muzychuk, Generalized table algebras, Israel Journal of Mathematics 114 (1999), 29-60.

[2] T. Beth, D. Jungnickel and H. Lenz, Design Theory, Cambridge University Press, Cambridge, 1986.

[3] A. Hanaki, Clifford theory for association schemes, Journal of Algebra 321 (2009), 16861695.

[4] A. Hanaki and M. Hirasaka, Theory of Hecke algebras to association schemes, SUT Journal of Mathematics 38 (2002), 61-66.

[5] A. Hanaki and P.-H. Zieschang, Representation theory of non-commutative association schemes of order 6, Oberwolfach Preprints (2010).

[6] D. G. Higman, Coherent configurations. I. Ordinary representation theory, Geometriae Dedicata 4 (1975), 1-32.

[7] Maxima.sourceforge.net, Maxima, a computer algebra system, version 5.21.1 (2010), http://maxima.sourceforge.net/. 
[8] P.-H. Zieschang, An Algebraic Approach to Association Schemes, Lecture Notes in Mathematics, Vol. 1628, Springer-Verlag, Berlin, 1996.

[9] P.-H. Zieschang, Theory of Association Schemes, Springer Monographs in Mathematics, Springer-Verlag, Berlin, 2005. 